\title{
PHOTOELECTRON EMISSION MICROSCOPY AND ITS APPLICATION TO THE STUDY OF POLYMER SURFACES
}

\author{
A. Cossy-Favre, J. Diaz, S. Anders, H. Padmore \\ Advanced Light Source, LBNL, 1, Cyclotron Road, Berkeley, CA 94720, USA \\ Y. LiU, M. Samant, J. Stöhr, H. Brown and T.P. Russell \\ IBM Almaden Research Center, 650 Harry Road, San Jose, CA 95120, USA
}

The X-ray photoelectron emission microscopy at the Advanced Light Source has a spatial resolution of 0.2 microns at an accelerating voltage of $12 \mathrm{kV}$. The tunability of the photon energy is used to provide chemical state information using near edge $\mathrm{X}$-ray absorption fine structure spectroscopy on the sub-micrometer scale. The homogeneity of thin films of polymer blends was studied for various film thicknesses. The polystyrene/poly vinylmethylether film of $194 \AA$ showed protrusions of $2-3 \mu \mathrm{m}$ diameter with an enriched polystyrene content while the polystyrene/polystyreneacrylonitrile $504 \AA$ thick films showed 5-6 $\mu \mathrm{m}$ segregated regions without any topological structure.

PACS numbers: 61.16.Ms, 73.61.Ph, 64.75.+g

\section{Introduction}

After the first photoelectron images taken by Brücke [1] in 1933, interest in the photoemission imaging technique increased dramatically when the UHV technology allowed the study of clean surfaces. The use of synchrotron light as the excitation source further opened the possibilities to develop the so-called X-ray photoelectron emission microscope (X-PEEM) [2-4] based on the near edge X-ray absorption fine structure (NEXAFS) [5] technique to obtain spectroscopic information on a sub-micron scale.

Here we present a study of phase segregation in thin films of polymer binary mixtures with X-PEEM. Phase segregation in polymer thin film mixtures confined between two surfaces has been the focus of much recent research [6-8]. The roughening at vacuum/polymer interface above the critical solution temperature of polymer blends was reported by Slawecki et al. [9] where they probed the topography and elasticity of the sample surfaces using variety of surface sensitive techniques, 
including scanning probe microscopy (AFM) and static secondary ion mass spectrometry. The correlation between the topography and polymer compositions were extracted indirectly from the friction and elasticity measurement with scanning probe microscopy. No measurements were performed to probe the morphology and at the same time detect the chemical composition of the surface. The advantage of $\mathrm{X}$-PEEM is that it can map both topography and chemical compositions of surfaces of polymer films with sub-micron lateral resolution and with exceptional surface depth sensitivity $(10 \mathrm{~nm})$. Further more, X-PEEM has much lower radiation damage to the polymer thin films in comparison with other electron microscopy techniques. This is shown in the study of phase separated polymer blends by Ade et al. [10]. Therefore, X-PEEM provides us a powerful tool for studying phase segregation of polymer thin films with capability to correlate the surface morphology and the polymer phase compositions. In this paper, we report the study of phase segregation in a thin film of polystyrene/polyvinylmethylether (PS/PVME) mixture, using X-PEEM to obtain combined topological and NEXAFS spectra, and the investigation of polystyrene/polystyrene-polyacrylonitrile (PS/PSAN) thin films using element specificity of NEXAFS.

\section{X-PEEM}

The instrument used at the Advanced Light Source (ALS) was built in the group of Tonner $[3,4,11]$. The optical system is designed on the basis of the tabulated properties of unipotential lenses measured by Rempfer [12] and consists of two electrostatic lenses, an accelerating objective and a projective lens giving a combined maximum magnification factor of 1000 . Resolution tests were out on $\mathrm{Cu}$ patterns deposited on a Si oxidized wafer using the $10 \% / 90 \%$ knifc edge resolution criteria. For an accelerating voltage of $12 \mathrm{kV}$ the resolution was $0.2 \mu \mathrm{m}$, limited primarily by the chromatic and spherical aberrations of the accelerating field.

\section{Polymers blends}

Two types of binary polymers blend thin films were produced by spin coating a toluene solution onto $\mathrm{HF}$ etched $\mathrm{Si}$ substrates and annealing for 3 hours at $170^{\circ} \mathrm{C}$ in $1 \times 10^{-6} \mathrm{~Pa}$ to prevent oxidative degradation. The thickness of the films was determined by ellipsometry. The X-PEEM measurements were taken on the undulator Beamline 7.0 at the ALS [13]. The photon flux on the sample was maintained below $4 \times 10^{11}$ photons/s (spot size $100 \times 100 \mu \mathrm{m}$ ) in order to minimize degradation of the polymers. The spectral resolution at $300 \mathrm{eV}$ was $\delta E=0.1 \mathrm{eV}$.

The first sample was a $194 \AA$ thick film of a mixture of $15 \%$ polystyrene (PS of $M w=96,000$ and $M w / M n=1.04$ ) and $85 \%$ polyvinylmethylether (PVME of $M w=84,000$ and $M w / M n=1.40$ ). Only the polystyrene polymer has benzene rings. The NEXAFS carbon $K$ edge absorption spectra of polystyrene shows $\pi^{*}$ resonances at $285 \mathrm{eV}[5]$ characteristic of the $\mathrm{C}=\mathrm{C}$ bonds in the benzene ring. An X-PEEM image taken at a photon energy of $270 \mathrm{eV}$ is shown in Fig. 1. Dark spots are $2-3 \mu \mathrm{m}$ diameter. A better understanding of the information contained in Fig. 1 is obtained by collecting NEXAFS spectra within four different regions of this sample. Regions 1 and 2 are defined inside the dark spots and regions 3 and 4 


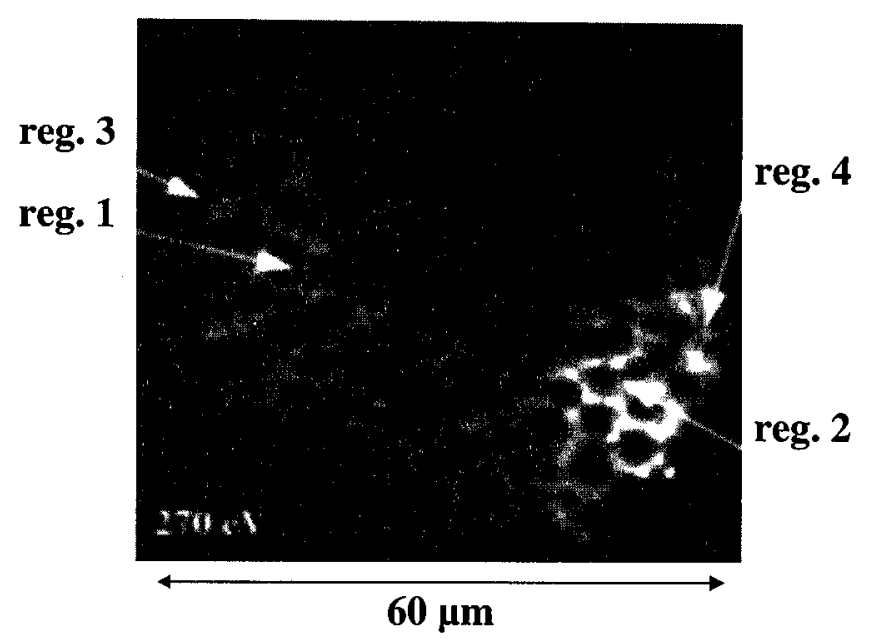

Fig. 1. Image of a $194 \AA$ thick blend of $15 \%$ PS $+85 \%$ PVME recorded at $270 \mathrm{eV}$. Field of view $60 \mu \mathrm{m}$.
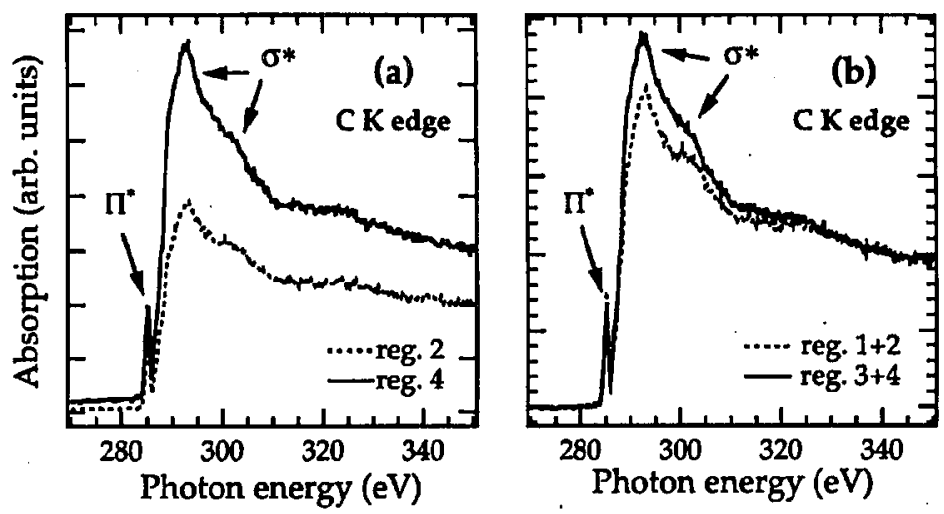

Fig. 2. NEXAFS carbon $K$ edge absorption spectra recorded in the regions 1 to 4 defined in Fig. 1. In part (a) the spectra are displayed as recorded without any normalization while in part (b) a constant background was subtracted below the absorption edge and they are normalized at $340 \mathrm{eV}$ to get the signal per atoms. In part (b) both signals of regions $1+2$ from the dark spots (protrusions) and both signal from the surrounding regions $3+4$ have been averaged.

are in the surrounding bright region. In Fig. 2a we have plotted the corresponding absorption spectra at the carbon $K$ edge. At $270 \mathrm{eV}$ most of the signal originates from the substrate $\mathrm{Si} L_{2,3}$ edge. Thus the observed contrast is due to variation in thickness of the coating with the bright regions corresponding to presence of thinner coating. The dark spots correspond to protrusions. Such protrusions were also observed on similar blends by Slawecki et al. [9] by means of atomic force microscopy. They reported an enriched concentration of PS within the protrusions. 
We confirm their findings by analyzing the normalized NEXAFS [5] spectra in Fig. 2b. Both regions (protrusions and surrounding) contain some PS as indicated by the presence of the $\pi^{*}$ resonance, but the concentration ratio of PS over PVME is higher within the protrusions as the ratio of the $\pi^{*}$ over $\sigma^{*}$ resonance is higher. The formation of the PS enriched protrusions during the annealing process is not accompanied by a total dewetting of blend in the region surrounding the protrusions. This example illustrates the possibility to obtain a topological information combined with the chemical distribution in the phase segregation of PS/PVME blends.

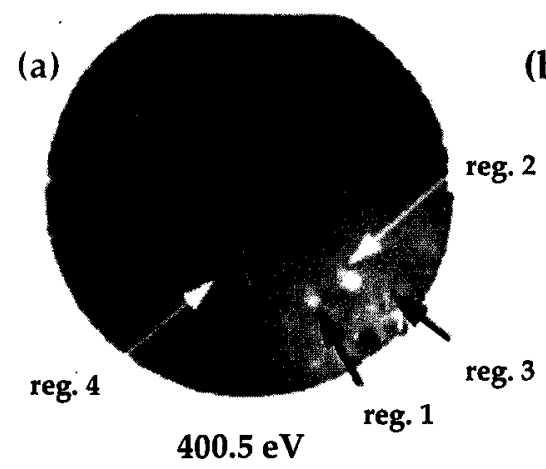

(b)

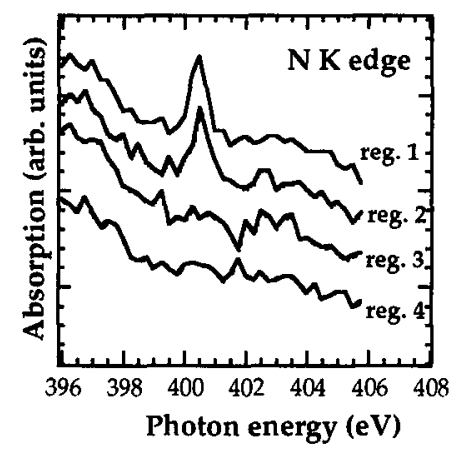

Fig. 3. (a) Image of a $504 \AA$ thick blend of $50 \%$ PS $+50 \%$ PSAN recorded at $400.5 \mathrm{eV}$. Field of view $60 \mu \mathrm{m}$. The two black spots on the right side of the image correspond to burned channels of the multichannel plate image intensifier. (b) NEXAFS nitrogen $K$ edge absorption spectra recorded in the regions 1 to 4 defined in part (a). Spectra not normalized and rigidly shifted along the intensity axis.

The second sample was a $504 \AA$ thick film composed of $50 \%$ polystyrene (PS of $M w=96,000$ and $M w / M n=1.04$ ) with $50 \%$ of polystyrene-polyacrylonitrile (PSAN of $M w=152,100$ and $M w / M n=2.03$ ) diblock copolymer. The weight percentage of the PS and PAN in the PSAN copolymer is $75 \%$ and $25 \%$ respectively. PSAN is differentiated from PS by the presence of a $N \equiv O$ along the polymeric chain. An image of this blend obtained at the nitrogen $K$ edge $(400.5 \mathrm{eV})$ is displayed in Fig. 3a showing some brighter regions of about 5-6 $\mu \mathrm{m}$ diameter. The NEXAFS spectra taken within these brighter regions (regions 1 and 2 on the picture) are displayed in Fig. $3 \mathrm{~b}$, confirming indeed the presence of nitrogen. In this case the X-PEEM analysis is sensitive to a pure chemical contrast. No domains were distinguished below the nitrogen $K$ edge. Other blends of PS/PVME and PS/PSAN of thickness $30 \AA$ were studied in the same way. We could not distinguish any sign of topological nor chemical segregation within our resolution limit.

A low intensity of the nitrogen $K$ edge absorption peak compared to the background signal in Fig. $3 \mathrm{~b}$ is due to radiation damage of the sample. In order to perform micro-NEXAFS studies on very sensitive materials such as polymers, one 
has to work at very low X-ray intensities. To improve the signal to noise ratio in the images the design of the microscope optics must be optimized to maximize its electron transmission function. To achieve a high electron transmission simultaneously with a high spatial resolution, our new X-PEEM will include an electrostatic hyperbolic electron mirror [14] to correct the chromatic and spherical aberration of the electrostatic lenses and of the accelerating field between the sample and the objective. In addition it will work at substantially higher voltage $(30 \mathrm{kV})$ and use a much more efficient YAG-cooled CCD detector instead of the present channel plate/phosphor/CCD combination currently used.

\section{Acknowledgments}

We are very grateful to J. Denlinger and A. Warwick for their contribution at different levels. This work was supported by the Director, Office of Energy Research, Office of Basic Energy Sciences, Materials Sciences Division of the U.S. Department of Energy, under contract No. DE-AC03-76SF00098. We are also very grateful to the Swiss National Science Foundation, the Societe Académique Vaudoise and the Ministerio de Educacion y Ciencia of Spain for their financial support.

\section{References}

[1] E. Brücke, Z. Phys. 86, 448 (1933).

[2] Ultramicroscopy 36 (1991).

[3] B.P. Tonner, G.R. Harp, J. Vacuum Sci. Technol. A 7, 1 (1989).

[4] B.P. Tonner, G.R. Harp, Rev. Sci. Instrum. 59, 853 (1988).

[5] J. Stöhr, NEXAFS Spectroscopy, Surface Science, Vol. 25, Springer, Berlin 1992.

[6] H. Tanaka, Europhys. Lett. 24, 665 (1993) and references therein.

[7] A.J. Liu, D.J. Durian, E. Herbolzheimer, S.A. Safran, Phys. Rev. Lett. 65, 1897 (1990).

[8] S. Reich, Y. Cohen, J. Polymer Sci. Polymer Phys. Ed. 19, 1255 (1981).

[9] T. Slawecki, A. Karim, S.K. Kumar, T.P. Russell, S.K. Satija, C.C. Han, Y. Liu, M.H. Rafailovich, J. Sokolov, R. Overney, submitted to Europhys. Lett.

[10] H. Ade, X. Zhang, S. Cameron, C. Costello, J. Kirz, S. Williams, Science 258, 972 (1992).

[11] D. Dunham, D.M. Desloge, G.F. Rempfer, W.P. Skoczyclas, T.B. Tonner, Nucl. Instrum. Methods Phys. Res. A 347, 441 (1994).

[12] G.F. Rempfer, J. Appl. Phys. 57, 2385 (1985).

[13] A. Warwick, P. Heimann, D. Mossessian, W. McKinney, H.A. Padmore, Rev. Sci. Instrum. 66, 2037 (1995).

[14] G.F. Rempfer, J. Appl. Phys. 67, 6027 (1990). 